\title{
On some two dimensional Volterra type linear integral equations with super-singularity
}

Nusrat Rajabov, Miklós Rontó, and Lutfia Rajabova 


\title{
ON SOME TWO DIMENSIONAL VOLTERRA TYPE LINEAR INTEGRAL EQUATIONS WITH SUPER-SINGULARITY
}

\author{
NUSRAT RAJABOV, MIKLÓS RONTÓ, AND LUTFIA RAJABOVA
}

[Received: December 22, 2002]

\begin{abstract}
Aвstract. In this paper the two dimensional linear Volterra type of integral equations containing singularity or super-singularity in the kernel function is considered. For different values of parameters in the integral equations existence theorems are proved for homogeneous and inhomogeneous equations.
\end{abstract}

Mathematics Subject Classification: 45E99

Keywords: two dimensional Volterra type integral equations, singularity, supersingularity, linear independent solutions

\section{Problem description}

Let $D$ denote the rectangle $D=\left\{(x, y): a<x<x_{0}, b_{0}<y<b\right\}, \Gamma_{1}=\{a<x<$ $\left.x_{0}, y=b\right\}, \Gamma_{2}=\left\{x=a, b_{0}<y<b\right\}$. In the domain $D$ we consider the following integral equation

$$
\begin{gathered}
U(x, y)+\lambda \int_{a}^{x} \frac{U(t, y)}{(t-a)^{\alpha}} d t-\mu \int_{y}^{b} \frac{U(x, s)}{(b-s)^{\beta}}+ \\
+\delta \int_{a}^{x} \frac{d t}{(t-a)^{\alpha}} \int_{y}^{b} \frac{U(t, s)}{(b-s)^{\beta}} d s(x, y)=f(x, y)
\end{gathered}
$$

where $\alpha>0, \beta>0$ and $\lambda, \mu, \delta$ are constants, the right-hand side function $f(x, y) \in$ $C(\bar{D})$ is a given function in $\bar{D}$, such that it vanishes on the lines $\Gamma_{1}, \Gamma_{2}$.

If the integral equation (1.1) has a solution and the corresponding integrals are convergent, then passing to the limit in (1.1) as $y \rightarrow b$ and $x \rightarrow a$, we obtain on $\Gamma_{2}$ and $\Gamma_{1}$ the following two one-dimensional Volterra type integral equations:

$$
\begin{aligned}
& U(a, y)-\mu \int_{y}^{b} \frac{U(a, s)}{(b-s)^{\beta}} d s=f(a, y), \\
& U(x, b)+\lambda \int_{a}^{x} \frac{U(t, b)}{(t-a)^{\alpha}} d t=f(x, b) .
\end{aligned}
$$


The equations (1.2) and (1.3) are studied in detail in [1], [2].

It should be noted that the integral equation (1.1) under the following restrictions for the pairs of parameters $\alpha<1, \beta<1 ; \alpha<1, \beta=1 ; \alpha=1, \beta<1$ and $\alpha>1, \beta>$ 1 was investigated in [3], [4].

In this paper the general solution of the integral equation (1.1) is constructed for the pairs $\alpha=1, \beta>1$ and $\alpha>1, \beta=1$. It will be established that for certain values of parameters $\lambda$ and $\mu$ the corresponding homogeneous integral equation (1.1) has an infinite number of linear independent solutions and for other values of $\lambda, \mu$ the homogeneous integral equation (1.1) has no other solutions than the trivial solution. Under some additional conditions it will be proved that the inhomogeneous integral equation (1.1) for certain values of $\lambda, \mu$ is solvable, and for some other values of $\lambda, \mu$ has a unique solution. In the case when $\delta=-\lambda \mu$ in (1.1), the solution can be found using a well known approach.

It seems to the authors that the integral equation (1.1) with super-singularity has not been studied yet.

\section{SiNGULARITY ON THE FIRST AND SUPER-SINGULARITY ON THE SECOND VARIABLE}

Theorem 2.1 Let us suppose that $\alpha=1, \beta>1, \mu>0, \lambda<0$, and $\delta=-\lambda \mu$ in integral equation (1.1). Moreover, assume that the behaviour of the right-hand side function $f(x, y) \in C(\bar{D})$ is determined by the following asymptotic formulae

$$
\begin{gathered}
f(x, y)=o\left[\exp \left(-\mu \omega_{b}^{\beta}(y)\right)(b-y)^{\gamma_{1}}\right], \gamma_{1}>\beta \\
\omega_{b}^{\beta}(y)=\left[(\beta-1)(b-y)^{\beta-1}\right]^{-1},
\end{gathered}
$$

as $y \rightarrow b$ and

$$
f(x, y)=o\left[(x-a)^{\gamma_{2}}\right], \gamma_{2}>|\lambda|,
$$

as $x \rightarrow a$.

Then the corresponding homogeneous integral equation (1.1) in the class of functions $C(\bar{D})$ has an infinite number of linear independent solutions. The non-homogeneous integral equation (1.1) is always solvable in $C(\bar{D})$ and its general solution containing four arbitrary functions is given by the following formula

$$
\begin{gathered}
U(x, y)=\exp \left[-\mu \omega_{b}^{\beta}(y)\right] \varphi_{1}(x)+(x-a)^{-\lambda} \psi_{1}(y)+ \\
+(x-a)^{-\lambda} \exp \left[-\mu \omega_{b}^{\beta}(y)\right] \int_{y}^{b} \exp \left[\mu \omega_{b}^{\beta}(s)\right] \psi_{2}(s) d s+ \\
+(x-a)^{-\lambda} \exp \left[-\mu \omega_{b}^{\beta}(y)\right] \int_{a}^{x}(t-a)^{\lambda} \varphi_{2}(t) d t+f(x, y)-
\end{gathered}
$$




$$
\begin{gathered}
-\lambda \int_{a}^{x}\left(\frac{t-a}{x-a}\right)^{\lambda} \frac{f(t, y)}{t-a} d t+\mu \int_{y}^{b} \frac{\exp \left[\mu\left(\omega_{b}^{\beta}(s)-\omega_{b}^{\beta}(y)\right)\right]}{(b-s)^{\beta}} f(x, s) d s- \\
-\lambda \mu \int_{a}^{x}\left(\frac{t-a}{x-a}\right)^{\lambda} \frac{d t}{t-a} \int_{y}^{b} \frac{\exp \left[\mu\left(\omega_{b}^{\beta}(s)-\omega_{b}^{\beta}(y)\right)\right]}{(b-s)^{\beta}} f(t, s) d s \equiv \\
\equiv T_{1, \beta}\left[\varphi_{1}(x), \varphi_{2}(x), \psi_{1}(y), \psi_{2}(y), f(x, y)\right],
\end{gathered}
$$

In (2.3) $\varphi_{j}(x), \psi_{j}(y), j=1,2$ are arbitrary functions defined on $\Gamma_{1}, \Gamma_{2}$, such that $\varphi_{j}(x) \in C\left(\bar{\Gamma}_{1}\right), \psi_{j}(y) \in C\left(\bar{\Gamma}_{2}\right)(j=1,2)$ satisfying the following conditions as $x \rightarrow$ $a, y \rightarrow b:$

$$
\begin{gathered}
\varphi_{1}(x)=o\left[(x-a)^{\gamma_{3}}\right], \gamma_{3}>0 \text { as } x \rightarrow a \\
\psi_{1}(y)=o\left[(b-y)^{\gamma_{4}}\right], \gamma_{4}>\beta-1 \text { as } y \rightarrow b \\
\varphi_{2}(x)=o\left[(x-a)^{\gamma_{5}} \exp \left(-\mu \omega_{b}^{\beta}(y)\right)\right], \gamma_{5}>|\lambda| \text { as } x \rightarrow a \\
\psi_{2}(y)=o\left[\exp \left(-\mu \omega_{b}^{\beta}(y)\right)(b-y)^{\gamma_{6}}\right], \gamma_{6}>\beta-1 \text { as } y \rightarrow b .
\end{gathered}
$$

Proof. The proof of Theorem 2.1 essentially uses the theory of partial differential equations of hyperbolic type with singular and super-singular lines [5], [6]. For the values of parameters $\alpha=1, \beta>1, \lambda<0, \mu>0$ let the integral equation (1.1) have a differentiable solution in the domain $D$. Then by differentiating (1.1) with respect to $x$ and $y$, we get the following equality

$$
\frac{\partial^{2} U}{\partial x \partial y}+\frac{\lambda}{x-a} \frac{\partial U}{\partial x}+\frac{\mu}{(b-y)^{\beta}} \frac{\partial U}{\partial x}-\frac{\delta}{(x-a)(b-y)^{\beta}} U=\frac{\partial^{2} f}{\partial x \partial y} .
$$

Thus we reduce the given problem to finding a solution of the so-called model type second order linear hyperbolic equation with one singular line on $\Gamma_{1}$ and one supersingular line on $\Gamma_{2}$. The theory of the model equation obtained above is constructed in [5-6]. According to [5-6], the solution of the model equation for $\lambda<0, \mu>0, \delta=$ $-\lambda \mu$ has the form:

$$
\begin{gathered}
U(x, y)=\exp \left[-\mu \omega_{b}^{\beta}(y)\right] \varphi_{1}(x)+(x-a)^{-\lambda} \psi_{1}(y)+ \\
+(x-a)^{-\lambda} \exp \left[-\mu \omega_{b}^{\beta}(y)\right] \int_{y}^{b} \exp \left[\mu \omega_{b}^{\beta}(s)\right] \psi_{2}(s) d s+ \\
+(x-a)^{-\lambda} \exp \left[-\mu \omega_{b}^{\beta}(y)\right] \int_{a}^{x}(t-a)^{\lambda} \varphi_{2}(t) d t-
\end{gathered}
$$




$$
-\int_{a}^{x}\left(\frac{t-a}{x-a}\right)^{\lambda} d t \int_{y}^{b} \exp \left[\mu\left(\omega_{b}^{\beta}(s)-\omega_{b}^{\beta}(y)\right)\right] \frac{\partial^{2} f}{\partial t \partial s} d s .
$$

Performing the necessary transformations in this expression and using the conditions of Theorem 2.1, we get the integral representation (2.3), which is obtained by supposing that $\frac{\partial^{2} f}{\partial x \partial y} \in C(\bar{D})$. By direct computation one can check that under the conditions of the theorem the solution having the form (2.3) satisfies the integral equation (1.1) even in the case when $f(x, y) \in C(\bar{D})$.

Theorem 2.2 Let us suppose that $\alpha=1, \beta>1, \mu<0, \lambda>0, \delta=-\lambda \mu$ in the integral equation (1.1) and $f(x, y) \in C(\bar{D})$. In addition, assume that on the lines $\Gamma_{1}, \Gamma_{2}$ the function $f(x, y) \in C(\bar{D})$ possesses the following asymptotic properties

$$
f(x, y)=o\left[(x-a)^{\gamma_{7}}\right], \gamma_{7}>0,
$$

as $x \rightarrow a$ and

$$
f(x, y)=o\left[(b-y)^{\gamma_{8}}\right], \gamma_{8}>\beta-1
$$

as $y \rightarrow b$.

Then on the one hand, the corresponding homogeneous integral equation (2.1) has only the trivial solution in the class of functions $C(\bar{D})$. On the other hand, the non-homogeneous integral equation (1.1) is always uniquely solvable in $C(\bar{D})$ and its solution is given by formula

$$
U(x, y)=T_{1, \beta}[0,0,0,0, f(x, y)] .
$$

Theorem 2.3. Let us assume that in the integral equation (1.1) $\alpha=1, \beta>1, \mu<$ $0, \lambda<0, \delta=-\lambda \mu$ and the asymptotic behaviour of the function $f(x, y) \in C(\bar{D})$ is determined according to (2.2) and (2.9) as $x \rightarrow a$ and as $y \rightarrow b$, respectively. Then the corresponding homogeneous integral equation $(1.1)$ in $C(\bar{D})$ has an infinite number of linear independent solutions. Moreover, the non-homogeneous equation (1.1) in $C(\bar{D})$ is also always solvable and its general solution contains two arbitrary functions defined on $\Gamma_{2}$ and given by the following formula

$$
U(x, y)=T_{1, \beta}\left[0,0, \psi_{1}(y), \psi_{2}(y), f(x, y)\right],
$$

where $\psi_{1}(y), \psi_{2}(y)$ arbitrary functions defined on $\Gamma_{2}$ such that $\psi_{1}(y)$ has the property (2.5) and $\psi_{2}(y)$ satisfy the condition

$$
\psi_{2}(y)=o\left[(b-y)^{\gamma_{9}}\right], \gamma_{9}>\beta-1 .
$$

as $y \rightarrow b$

Theorem 2.4. If in the integral equation (1.1) $\alpha=1, \beta>1, \mu>0, \lambda>$ $0, \delta=-\lambda \mu$ and the right hand side function $f(x, y) \in C(\bar{D})$ vanishing on $\Gamma_{2}$ has the asymptotic property (2.1) as $y \rightarrow b$, then the corresponding homogeneous integral 
equation (1.1) in $C(\bar{D})$ is always solvable. In this case the general solution contains two arbitrary functions defined on $\Gamma_{1}$ and can be written in the form

$$
U(x, y)=T_{1, \beta}\left[\varphi_{1}(x), \varphi_{2}(x), 0,0, f(x, y)\right],
$$

where $\varphi_{1}(x), \varphi_{2}(x)$ are arbitrary functions defined on $\Gamma_{1}$ such that $\varphi_{1}(x)$ satisfy condition (2.4) as $x \rightarrow$ a and for $\varphi_{2}(x) \in C\left(\bar{\Gamma}_{1}\right)$ holds the equality

$$
\varphi_{2}(x)=o\left[(x-a)^{\gamma_{10}}\right], \gamma_{10}>0 .
$$

as $x \rightarrow a$.

Remark 2.1. Under the conditions of Theorem 2.1 the solution of the form (2.3) vanishes on $\Gamma_{1}, \Gamma_{2}$ according to the following asymptotic formula

$$
\begin{gathered}
U(x, y)=o\left[(x-a)^{\gamma_{11}}\right], \gamma_{11}>0, \text { as } x \rightarrow a, \\
U(x, y)=o\left[(b-y)^{\gamma_{12}}\right], \gamma_{12}>\beta-1, \text { as } y \rightarrow b .
\end{gathered}
$$

Remark 2.2. If the conditions of Theorem 2.2 hold, then the solution of the form (2.10) vanishes on $\Gamma_{1}, \Gamma_{2}$ satisfying the following relations

$$
\begin{gathered}
U(x, y)=o\left[(b-y)^{\gamma_{13}}\right], \gamma_{13}>\beta-1, \\
U(a, y)=f(o, y)-f(o, y)+\mu \int_{y}^{b} \frac{\exp \left[\mu\left(\omega_{b}^{\beta}(s)-\omega_{b}^{\beta}(y)\right)\right]}{(b-s)^{\beta}} f(a, s) d s- \\
-\mu \int_{y}^{b} \frac{\exp \left[\mu\left(\omega_{b}^{\beta}(s)-\omega_{b}^{\beta}(y)\right)\right]}{(b-s)^{\beta}} f(a, s) d s=0
\end{gathered}
$$

as $x \rightarrow a, y \rightarrow b$.

Remark 2.3. Under the conditions of Theorem 2.3 the solution of the form (2.11) vanishes on $\Gamma_{1}, \Gamma_{2}$ satisfying the following relations as $x \rightarrow a, y \rightarrow b$

$$
\begin{gathered}
U(x, y)=o\left[(x-a)^{|\lambda|}\right], \text { as } x \rightarrow a, \\
U(x, y)=o\left[(b-y)^{\beta}\right], \text { as } y \rightarrow b .
\end{gathered}
$$

Remark 2.4. If the conditions of Theorem 2.4 are satisfied, then the solution (2.13) vanishes on $\Gamma_{1}, \Gamma_{2}$ such that the following asymptotic formulae hold as $x \rightarrow a, y \rightarrow b$

$$
\begin{gathered}
U(x, y)=o\left[(x-a)^{\gamma_{14}}\right], \gamma_{14}>0, \text { as } \quad x \rightarrow a, \\
U(x, y)=o\left[\exp \left(-\mu \omega_{b}^{\beta}(y)\right)\right], \quad \text { as } \quad y \rightarrow b .
\end{gathered}
$$




\section{SUPERSINGULARITY ON THE FIRST AND SINGULARITY ON THE SECOND VARIABLE}

Theorem 3.1. Let us assume that in the integral equation (1.1) $\alpha>1, \beta=1, \mu>$ $0, \lambda<0, \delta=-\lambda \mu$, and the right hand side function $f(x, y) \in C(\bar{D})$ satisfies the following asymptotic relations

$$
f(x, y)=o\left[\exp \left(\lambda \omega_{a}^{\alpha}(x)\right)(x-a)^{\gamma_{15}}\right], \quad \gamma_{15}>\alpha-1
$$

as $x \rightarrow$ and

$$
f(x, y)=o\left[(b-y)^{\gamma_{16}}\right], \gamma_{16}>\mu
$$

as $y \rightarrow b$.

Then the corresponding homogeneous integral equation (1.1) in $C(\bar{D})$ has an infinite number of linear independent solutions. Besides, the non-homogeneous integral equation (1.1) in $C(\bar{D})$ is always solvable and its general solution containing four arbitrary functions defined on $\Gamma_{1}, \Gamma_{2}$ has the form

$$
\begin{gathered}
U(x, y)=(b-y)^{\mu} \varphi_{1}(x)+\exp \left[\lambda \omega_{a}^{\alpha}(x)\right] \psi_{1}(y)+ \\
+(b-y)^{\mu} \exp \left[\lambda \omega_{a}^{\alpha}(x)\right] \int_{y}^{b}(b-s)^{-\mu} \psi_{2}(s) d s+(b-y)^{\mu} \exp \left[\lambda \omega_{a}^{\alpha}(x)\right] \times \\
\times \int_{x}^{a} \exp \left[-\lambda \omega_{a}^{\alpha}(t)\right] \varphi_{2}(t) d t+f(x, y)-\lambda \int_{a}^{x} \frac{\exp \left[\lambda\left(\omega_{a}^{\alpha}(x)-\omega_{a}^{\alpha}(t)\right)\right]}{(t-a)^{\alpha}} f(t, y) d t+ \\
+\mu \int_{y}^{b}\left(\frac{b-y}{b-s}\right)^{\mu} \frac{f(x, s)}{b-s} d s-\mu \lambda \int_{y}^{b}\left(\frac{b-y}{b-s}\right)^{\mu} \frac{d s}{b-s} \times \\
\times \int_{a}^{x} \frac{\exp \left[\lambda\left(\omega_{a}^{\alpha}(x)-\omega_{a}^{\alpha}(t)\right)\right]}{(t-a)^{\alpha}} f(t, s) d s \equiv \\
\equiv T_{\alpha, 1}\left[\varphi_{1}(x), \varphi_{2}(x), \psi_{1}(y), \psi_{2}(y), f(x, y)\right]
\end{gathered}
$$

where $\omega_{a}^{\alpha}(x)=\left[(\alpha-1)(x-a)^{\alpha-1}\right]^{-1}$, and the arbitrary functions $\varphi_{j}(x) \in C\left(\bar{\Gamma}_{1}\right)$ and $\psi_{j}(y) \in C\left(\bar{\Gamma}_{2}\right), j=1,2$ satisfy the following asymptotic relations as $x \rightarrow a, y \rightarrow b$

$$
\begin{aligned}
\varphi_{1}(x)= & o\left[(x-a)^{\gamma_{17}} \exp \left(\lambda \omega_{a}^{\alpha}(x)\right)\right], \gamma_{17}>\alpha-1, \text { as } x \rightarrow a, \\
& \psi_{1}(y)=o\left[(b-y)^{\gamma_{18}}\right], \gamma_{18}>0, \text { as } y \rightarrow b, \\
\varphi_{2}(x)= & o\left[\exp \left(\lambda \omega_{a}^{\alpha}(x)\right)(x-a)^{\gamma_{19}}\right], \gamma_{19}>\alpha-1, \text { as } x \rightarrow a, \\
& \psi_{2}(y)=o\left[(b-y)^{\gamma_{20}}\right], \gamma_{20}>\mu, \text { as } y \rightarrow b .
\end{aligned}
$$

From the integral representation (3.3) it follows: 
Remark 3.1. If the conditions of Theorem 3.1 are valid, then any solution of the integral equation (1.1) vanishes on $\Gamma_{1}$ and $\Gamma_{2}$ as $x \rightarrow a, y \rightarrow b$ in the following manner

$$
\begin{gathered}
U(x, y)=o\left[(x-a)^{\gamma_{21}}\right], \gamma_{21}>\alpha-1, \text { as } \quad x \rightarrow a, \\
U(x, y)=o\left[(b-y)^{\gamma_{22}}\right], \gamma_{22}>0, \text { as } \quad y \rightarrow b .
\end{gathered}
$$

Theorem 3.2. Let us assume that $\alpha>1, \beta=1, \mu<0, \lambda>0, \delta=-\lambda \mu$ hold in the integral equation (1.1) and the function $f(x, y) \in C(\bar{D})$ has the asymptotic behaviour

$$
f(x, y)=o\left[(x-a)^{\gamma_{23}}\right], \gamma_{23}>\alpha-1,
$$

as $x \rightarrow$ and

$$
f(x, y)=o\left[(b-y)^{\gamma_{24}}\right], \gamma_{24}>0
$$

as $y \rightarrow b$.

Then the corresponding homogeneous integral equation (1.1) in $C(\bar{D})$ has only the trivial solution, while the inhomogeneous equation (1.1) is uniquely solvable in $C(\bar{D})$. The solution is given by the formula

$$
U(x, y)=T_{\alpha, 1}[0,0,0,0, f(x, y)] .
$$

From Theorem 3.2 it follows:

Remark 3.2. Under the conditions of Theorem 3.2 any solution of the integral equation (1.1) vanishes on $\Gamma_{1}$ and $\Gamma_{2}$ having the asymptotic behaviour

$$
U(x, y)=o\left[(x-a)^{\gamma_{25}}\right], \gamma_{25}>\alpha-1,
$$

as $x \rightarrow a$ and

$$
\begin{gathered}
U(x, b)=f(x, b)-\lambda \int_{a}^{x} \frac{\exp \left[\lambda\left(\omega_{a}^{\alpha}(x)-\omega_{a}^{\alpha}(t)\right)\right]}{(t-a)^{\alpha}} f(t, b) d t- \\
-f(x, b)+\lambda \int_{a}^{x} \frac{\exp \left[\lambda\left(\omega_{a}^{\alpha}(x)-\omega_{a}^{\alpha}(t)\right)\right]}{(t-a)^{\alpha}} f(t, b) d t=0
\end{gathered}
$$

as $y \rightarrow b$.

Theorem 3.3. Let us suppose that in the integral equation (1.1) the relations $\alpha>1, \beta=1, \lambda<0, \mu<0, \delta=-\lambda \mu$ hold and the function $f(x, y) \in C(\bar{D})$ fulfils the following asymptotic relations

$$
f(x, y)=o\left[(x-a)^{\gamma_{26}}\right], \gamma_{26}>\alpha-1, \text { as } x \rightarrow a
$$

and

$$
f(x, y)=o\left[(b-y)^{\gamma_{27}}\right], \gamma_{27}>|\mu| \text { as } y \rightarrow b
$$

Then the homogeneous integral equation (1.1) in $C(\bar{D})$ has an infinite number of linear independent solutions. Moreover, the non-homogeneous equation (1.1) in $C(\bar{D})$ 
is always solvable and its general solution contains two arbitrary functions and has the form

$$
U(x, y)=T_{\alpha, 1}\left[0,0, \psi_{1}(y), \psi_{2}(y), f(x, y)\right],
$$

where $\psi_{1}(y), \psi_{2}(y)$ are arbitrary functions such that $\psi_{1}(y)$ satisfy the condition (3.5) while for the function $\psi_{2}(y)$ holds the following relation

$$
\psi_{2}(y)=o\left[(b-y)^{\gamma_{28}}\right], \gamma_{28}>0
$$

as $y \rightarrow b$.

From the integral representation (3.9) it follows:

Remark 3.3. If the conditions of Theorem 3.3 hold then any solution of the integral equation (1.1) vanishes on $\Gamma_{1}, \Gamma_{2}$ with the following asymptotic behaviour

$$
\begin{gathered}
U(x, y)=o\left[\exp \left(\lambda \omega_{a}^{\alpha}(x)\right)\right], \text { as } \quad x \rightarrow a, \\
U(x, y)=o\left[(b-y)^{\gamma_{29}}\right], \gamma_{29}>0 .
\end{gathered}
$$

In the case when the parameters $\lambda$ and $\mu$ are positive, we have the assertion:

Theorem 3.4. Let us assume that in the integral equation (1.1) the relations $\alpha>$ $1, \beta=1, \lambda>0, \mu>0, \delta=-\lambda \mu$, hold and for the function $f(x, y) \in C(\bar{D})$ the following asymptotic relations

$$
f(x, y)=o\left[(x-a)^{\gamma_{30}} \exp \left[-\lambda \omega_{a}^{\alpha}(x)\right], \gamma_{30}>\alpha-1,\right.
$$

as $x \rightarrow a$ and

$$
f(x, y)=o\left[(b-y)^{\gamma_{31}}\right], \gamma_{31}>\mu, \text { as } \quad y \rightarrow b
$$

hold. Then the homogeneous integral equation (1.1) has an infinite number of linear independent solutions in the class of functions $C(\bar{D})$. The non-homogeneous equation (1.1) is also solvable in $C(\bar{D})$. The general solution contains two arbitrary functions

$$
U(x, y)=T_{\alpha, 1}\left[\varphi_{1}(x), \varphi_{2}(x), 0,0, f(x, y)\right],
$$

where $\varphi_{1}(x), \varphi_{2}(x)$ are arbitrary functions satisfying the following conditions

$$
\begin{array}{ll}
\varphi_{1}(x)=o\left[(x-a)^{\gamma_{32}}\right], & \gamma_{32}>\alpha-1, \\
\varphi_{2}(x)=o\left[(x-a)^{\gamma_{33}}\right], & \gamma_{33}>\alpha-1
\end{array}
$$

as $x \rightarrow a$.

From Theorem 3.4 it follows:

Remark 3.4. Under the conditions of Theorem 3.4 any solution of the integral equation (1.1) vanishes on $\Gamma_{1}, \Gamma_{2}$ with the following asymptotic properties

$$
\begin{gathered}
U(x, y)=o\left[(x-a)^{\gamma_{34}}\right], \gamma_{34}>\alpha-1, \text { as } \quad x \rightarrow a, \\
U(x, y)=o\left[(b-y)^{\gamma_{\mu}}\right], \text { as } y \rightarrow b .
\end{gathered}
$$




\section{SERIES REPRESENTATION OF THE SOLUTION WITH RESPECT TO ONE OF THE VARIABLES}

It is easy to see that in the case when $\delta \neq-\lambda \mu$ the problem of finding the solution of the integral equation (1.1) is reduced to the solution of this equation with $\lambda=$ $0, \mu=0$.

For example in the case when $\alpha>1, \beta=1, \mu>0, \lambda<0, \delta \neq-\lambda \mu$, finding the solution of equation (1.1) is reduced to studying the solution of the following integral equation

$$
V(x, y)+\delta_{1} \int_{a}^{x} \frac{d t}{(t-a)^{\alpha}} \int_{y}^{b} \frac{V(t, s)}{b-s} d s=G(x, y),
$$

where $\delta_{1}=\delta+\lambda \mu$,

$$
\begin{gathered}
V(x, y)=(b-y)^{-\mu} \exp \left[-\lambda \omega_{a}^{\alpha}(x)\right] U(x, y) \\
G(x, y)=(b-y)^{-\mu} \psi_{1}(y)+\exp \left[-\lambda \omega_{a}^{\alpha}(x)\right] \varphi_{1}(x)+ \\
+\int_{a}^{x} \exp \left(-\lambda \omega_{a}^{\alpha}(t)\right) \varphi_{2}(t) d t+\int_{y}^{b}(b-s)^{-\mu} \psi_{2}(s) d s+ \\
+(b-y)^{-\mu} \exp \left[-\lambda \omega_{a}^{\alpha}(x)\right] f(x, y)-\lambda(b-y)^{-\mu} \times \\
\times \int_{a}^{x} \frac{\exp \left[-\lambda \omega_{a}^{\alpha}(t)\right] f(t, y)}{(t-a)^{\alpha}} d t+\mu \exp \left[-\lambda \omega_{a}^{\alpha}(x)\right] \times \\
\times \int_{y}^{b} \frac{f(x, s) d s}{(b-s)^{\mu+1}}-\lambda \mu \int_{y}^{b} \frac{d s}{(b-s)^{\mu+1}} \int_{a}^{x} \frac{\exp \left[-\lambda \omega_{a}^{\alpha}(t)\right]}{(t-a)^{\alpha}} f(t, s) d s,
\end{gathered}
$$

$\varphi_{j}(x), \psi_{j}(y), j=1,2$ are arbitrary functions defined on $\Gamma_{1}, \Gamma_{2}$ such that $\varphi_{j}(x) \in$ $C\left(\bar{\Gamma}_{1}\right), \psi_{j}(y) \in C\left(\bar{\Gamma}_{2}\right), j=1,2$ and these functions satisfy the conditions (3.4)-(3.6) as $x \rightarrow a, y \rightarrow b$.

Let us search the solution of the integral equation (4.1) in the following form

$$
V(x, y)=\sum_{k=0}^{\infty}(b-y)^{k+\delta} V_{k}(x), \delta>0,
$$

where $V_{k}(x)$ are unknown functions.

Suppose that the function $G(x, y)$ has the following series representation

$$
G(x, y)=\sum_{k=0}^{\infty}(b-y)^{k+\delta} G_{k}(x),
$$

where $G_{k}(x)$ are given functions. Moreover, assume that the series (4.3) converge absolutely and uniformly. 
In this case in finding the solutions of the two-dimensional integral equation (4.1) one should determine the solutions of the following one-dimensional Volterra type integral equations

$$
V_{k}(x)+\frac{\delta_{1}}{k+\delta} \int_{a}^{x} \frac{V_{k}(t) d t}{(t-a)^{\alpha}}=G_{k}(x),
$$

$k=0,1,2, \ldots$, which were investigated in [1-2] in detail.

Using the technique from [1-2] for solving (4.4), we find that

$$
\begin{gathered}
V_{k}(x)=\exp \left[\frac{\delta_{1}}{k+\delta} \omega_{a}^{\alpha}(x)\right] c_{k}+G_{k}(x)- \\
-\frac{\delta_{1}}{k+\delta} \int_{a}^{x} \frac{\exp \left[+\frac{\delta_{1}}{k+\delta}\left(\omega_{a}^{\alpha}(x)-\omega_{a}^{\alpha}(t)\right)\right]}{(t-a)^{\alpha}} G_{k}(t) d t,
\end{gathered}
$$

where $c_{0}, c_{1}, c_{2}, \ldots$ are arbitrary constants.

The solutions of the form (4.5) were obtained, supposing that $\delta_{1}<0$ and the functions $G_{k}(x) \in C\left(\bar{\Gamma}_{0}\right)$ vanish in the point $x=c$ having the following asymptotic behaviour

$$
G_{k}(x)=o\left[\exp \left(\frac{\delta_{1}}{k+\delta} \omega_{a}^{\alpha}(x)\right)(x-a)^{\gamma_{35}}\right],
$$

$\gamma_{35}>\alpha-1, k=0,1,2, \ldots$

In this case, we have the following assertion.

Theorem 4.1. Let us assume that in the integral equation (4.1) relations $\alpha>$ $1, \mu>0, \lambda<0, \delta_{1}=\delta+\lambda \mu \neq 0, \delta_{1}<0 \quad(\delta<|\lambda| \mu)$ hold and moreover for the function $G(x, y)$ the series representation (4.3) holds, where $G_{k}(x)$ are given functions vanishing on $\Gamma_{1}$ with the asymptotic behaviour (4.6), where $\delta>0$. Then the corresponding homogeneous integral equation (4.1) in the class of functions having the series representation of the form (4.2) has an infinite number of linear independent solutions of the form

$$
V_{k}(x, y)=\exp \left[\frac{\delta_{1}}{k+\delta} \omega_{a}^{\alpha}(x)\right](b-y)^{k+\delta}, \quad k=0,1, \ldots, \delta>0 .
$$

The non-homogeneous integral equation (4.1) in the class of functions having the series form (4.2) is always solvable and this solution is given by the following formula

$$
\begin{aligned}
& V(x, y)=\sum_{k=0}^{\infty} \exp \left[\frac{\delta_{1}}{k+\delta} \omega_{a}^{\alpha}(x)\right](b-y)^{k+\delta} c_{k}+G(x, y)- \\
& -\sum_{k=0}^{\infty} \frac{\delta_{1}}{k+\delta}\left(\int_{a}^{x} \frac{\exp \left[\frac{\delta_{1}}{k+\delta}\left(\omega_{a}^{\alpha}(x)-\omega_{a}^{\alpha}(t)\right)\right]}{(t-a)^{\alpha}} G_{k}(t) d t\right)(b-y)^{k+\delta},
\end{aligned}
$$

where $c_{k}(k=0,1,2, \ldots)$ are arbitrary constants. 
Theorem 4.2. Let us suppose that in the equation (1.1) $\alpha>1, \beta=1, \mu>$ $0, \lambda<0, \delta_{1}=\delta+\lambda \mu \neq 0, \delta_{1}>0(\delta>|\lambda| \mu)$ and the function $G(x, y)$ is presented in the form (4.3), where $G_{k}(x)$ are given functions such that $G_{k}(x) \in C\left(\bar{\Gamma}_{1}\right)$. Then the corresponding homogeneous integral equation (4.1) is not solvable in the class of functions having the series representation of the form (4.2). In this case the nonhomogeneous integral equation (4.1) has a unique solution of the series form (4.2), which is given by the following formula

$$
V(x, y)=G(x, y)-\int_{a}^{x} \frac{G_{1}(x, y, t) d t}{(t-a)^{\alpha}},
$$

where

$$
G_{1}(x, y, t)=\sum_{k=0}^{\infty}(b-y)^{k+\delta} \frac{\delta_{1}}{k+\delta} \exp \left[\frac{\delta_{1}}{k+\delta}\left(\omega_{a}^{\alpha}(x)-\omega_{a}^{\alpha}(t)\right)\right] G_{k}(t) .
$$

Remark 3.5. If the conditions of Theorem 4.1 are met, then the solution having the series form (4.2) vanishes on $\Gamma_{1}, \Gamma_{2}$ and its behaviour is determined by the following asymptotic formula

$$
V(x, y)=o\left[\exp \left(-\varepsilon \omega_{a}^{\alpha}(x)\right)\right],
$$

as $x \rightarrow a$, where $\varepsilon$ is a sufficiently small positive constant satisfying the conditions $\varepsilon<\frac{\left|\delta_{1}\right|}{\delta+k}$ and

$$
V(x, y)=o\left[(b-y)^{\gamma_{36}}\right], \gamma_{36}>0, \text { as } \quad y \rightarrow b .
$$

Remark 3.6. Under the conditions of Theorem 4.2, the solution of the series form (4.2) vanishes also and its asymptotic behaviour is determined from the following equality

$$
V(0, y)=G(0, y)-G(0, y)=0, \text { as } \quad x \rightarrow a,
$$

i.e.,

$$
V(x, y)=o\left((b-y)^{\gamma_{37}}\right), \gamma_{37}>0, \text { as } \quad y \rightarrow b .
$$

The proof of the statements above can be carried out by using the technique proposed in $[5,6]$.

Acknowledgement. The research of the first and second authors was supported in part by the Hungarian NATO Science and Technology Fellowship Programme through grants 2041/NATO/2001 and 2042/NATO/2001, respectively.

\section{REFERENCES}

[1] Rajabov, N.: General Volterra type integral equations with left and right fixed singular and super-singular point in kernel, News Academy of Science the Republic of Tajikistan, Department Physics, Mathematics, Chemistry and Geology Sciences, 1, (2001), 30-46. (in Russian)

[2] Rajabov, N.: On Volterra type integral equations, Russian Doklady Acad. Sciences, 383(3), (2002), 314-317. 
[3] Rajabov, N. and Rajabova, L.: On a class of two dimensional linear Volterra type integral equations with boundary fixed singular kernels, ISAAC Conference on Complex Analysis, Differential Equations and Related Topics, September 17-21, 2002, Yerevan, Armenia, Book of Abstracts, $52-53$.

[4] Rajabov, N. and Rajabova, L.: An explicit solution to a class of two dimensional Volterra type linear integral equations with fixed boundary singular kernels, ICM 2002, Beijing 2002, August 20-28, Abstract of Short Communications and Poster Session, 229-229.

[5] Rajabov, N.: Integral representation and boundary value problems for differential equations with singular lines and singular surfaces, Vol. 4, Dushanbe, 1986. (in Russian)

[6] Rajabov, N.: An Introduction to the Theory of Partial Differential Equations with Super-Singular Coefficients, Tehran University Publications, Tehran, 1997.

\section{Authors' Addresses}

\section{Nusrat Rajabov:}

Presidium of the Academy of Sciences of Tajikistan, 734025 Dushanbe, Rudaki Ave. 33, Tajikistan E-mail address: nusrat@ac.tajik.net

\section{Miklós Rontó:}

Institute of Mathematics, University of Miskolc, 3515 Miskolc-Egyetemváros, Hungary

E-mail address: matronto@gold.uni-miskolc.hu

\section{Lutfia Rajabova:}

Tajik Technical University, 734025 Dushanbe, Acad. Rajabov Ave. 10, Tajikistan

E-mail address: n.rajabov@tajik.net 